\title{
Cryopreservation of unrelated donor hematopoietic stem cells: the right answer for transplantations during the COVID-19 pandemic?
}

\author{
Jesus Fernandez-Sojo ${ }^{1}{ }^{1} \cdot$ Carmen Azqueta $^{1} \cdot$ Elena Valdivia $^{1} \cdot$ Lluis Martorell $^{1} \cdot$ Laura Medina-Boronat $^{1}$ • \\ Nuria Martínez-Llonch ${ }^{1}$ - Silvia Torrents ${ }^{1} \cdot$ Margarita Codinach $^{1} \cdot$ Carme Canals $^{1} \cdot$ Izaskun Elorza $^{2} \cdot$ Rocio Parody $^{3}$. \\ Rodrigo Martino (iD ${ }^{4} \cdot$ Maria Trabazo (D) $^{5} \cdot$ Cristina Díaz de Heredia $^{6}{ }^{6}$ Christelle Ferra (iD $^{7} \cdot$ David Valcárcel $^{8}$. \\ Mónica Linares ${ }^{9} \cdot$ Águeda Ancochea ${ }^{10}$. Enric García-Rey ${ }^{11}$ • Nadia García-Muñoz ${ }^{12} \cdot$ Laura Medina $^{13}$. \\ Nerea Castillo $^{14} \cdot$ Enric Carreras $^{15} \cdot$ Juliana Villa $^{15} \cdot$ Sergio Querol $^{1}{ }^{1}$
}

Received: 27 November 2020 / Revised: 16 May 2021 / Accepted: 26 May 2021 / Published online: 14 June 2021

(c) The Author(s), under exclusive licence to Springer Nature Limited 2021

\begin{abstract}
Cryopreservation was recommended to ensure continuity of unrelated donor (UD) hematopoietic stem cell transplantation (HSCT) during COVID-19 pandemic. However, its impact on clinical outcomes and feasibility was not well known. We compared 32 patients who underwent UD HSCT using cryopreserved peripheral blood stem cells (PBSC) during the COVID-19 pandemic with 32 patients who underwent UD HSCT using fresh PBSC in the previous period. Median neutrophil engraftment was 17.5 and 17.0 days with cryopreserved and fresh grafts, respectively. Non-significant delays were found in platelet recovery days $(25.5$ versus $19.0 ; P=0.192)$ and full donor chimerism days $(35.0$ and $31.5 ; P=0.872)$ using cryopreserved PBSC. The rate of acute graft-versus-host disease at 100 days was $41 \%$ (95\% CI [21-55\%]) in cryopreserved group versus $31 \%(95 \% \mathrm{CI}[13-46 \%])$ in fresh group $(P=0.380)$. One-hundred days progression-relapse free survival and overall survival did not differ significantly. During COVID-19 pandemic, six frozen UD donations were not transfused and logistical and clinical issues regarding cryopreservation procedure, packaging, and transporting appeared. In summary, UD HSCT with cryopreserved PBSC was safe during this challenging time. More efforts are needed to ensure that all frozen grafts are transplanted and cryopreservation requirements are harmonized.
\end{abstract}

Jesus Fernandez-Sojo

jefernandez@bst.cat

Banc de Sang i Teixits, Barcelona, Spain

2 Pediatric Hematology Department, Hospital Sant Joan de Déu, Barcelona, Spain

3 Adult Hematology Department, Institut Catala d'OncologiaHospitalet, Barcelona, Spain

4 Adult Hematology Department, Hospital Universitari de la Santa Creu i Sant Pau, Barcelona, Spain

5 Pediatric Hematology Department, Hospital Universitari de la Santa Creu i Sant Pau, Barcelona, Spain

6 Pediatric Hematology Department, Hospital Universitari Vall d'Hebron, Barcelona, Spain

7 Adult Hematology Department, Institut Català d'OncologiaBadalona, Barcelona, Spain
8 Adult Hematology Department, Hospital Universitari Vall d'Hebron, Barcelona, Spain

9 Banc de Sang i Teixits, Hospital Universitari Vall d'Hebron, Barcelona, Spain

10 Banc de Sang i Teixits, Hospital Universitari Germans Trias i Pujol, Badalona, Spain

11 Banc de Sang i Teixits, Hospital Sant Joan de Déu, Barcelona, Spain

12 Banc de Sang i Teixits, Hospital Universitari de Bellvitge, H. Llobregat, Barcelona, Spain

13 Banc de Sang i Teixits, Hospital Universitari de la Santa Creu i Sant Pau, Barcelona, Spain

14 Banc de Sang i Teixits, Hospital Mutua de Terrassa, Terrassa, Barcelona, Spain

15 Spanish Bone Marrow Donor Registry, Josep Carreras Foundation and Leukemia Research Institute, Barcelona, Catalunya, Spain 


\section{Introduction}

Unrelated donor (UD) hematopoietic stem cells (HSC) are usually freshly transported away from transplant centers (TCs) by door-to-door courier services shortly after collection. This practice usually allows grafts to be transfused without the need for a cryopreservation step [1], and is enabled by the adequate provision of donor products through the national registries, under the support of the World Marrow Donor Association.

The emergence of COVID-19 caused by SARS-CoV-2 and the subsequent evolution to a global pandemic significantly disrupted graft transportation within and between countries. Suddenly, transit restrictions for people and goods made it difficult to guarantee the timely delivery of fresh UD HSC, which is a requirement for successful transplantation [1]. Moreover, the concern that the donor could be at risk of community-acquired infection once the patients had started the conditioning procedure led professional societies to recommend immediately the cryopreservation of grafts [2-4]. However, evidence about the feasibility of cryopreservation prior to UD HSC was scarce, since most of the literature has approached this topic within the context of match-related donors (MRD) [5-9], where logistics and time from collection to cryopreservation are perfectly controlled, and the few studies available have yielded controversial results $[5,10,11]$.

The aim of the present study was to retrospectively investigate the clinical outcomes, namely myeloid engraftment, full donor chimerism, acute graft-versus-host disease (GVHD), preemptive therapy on cytomegalovirus (CMV) disease, progression-relapse-free survival (PFS), and overall survival (OS), of patients who underwent UD hematopoietic stem cell transplantation (HSCT) with cryopreserved peripheral blood stem cells (PBSC) during COVID-19 pandemic, and compare them with those of patients who had undergone UD HSCT with fresh PBSC in the immediately previous period, as well as to identify frozen grafts not used, and logistical and clinical issues related to the cryopreservation of UD HSC during the COVID-19 pandemic.

\section{Materials and methods}

\section{Donors and patients}

All donors and recipients provided written informed consent prior to collection and transplantation. The Vall d'Hebron University Hospital's Ethics Committee reviewed and approved the study [CEIC code PR(AG)355/2020]. Eligibility criteria for patients were: (i) first allo-HSCT, (ii) nonmanipulated UD PBSC as graft source, (iii) no presence of donor-specific anti-human leukocyte antigen (HLA) antibodies (DSA), and (iv) partial or complete disease remission status at the time of the transplantation procedure. All consecutive patients meeting these criteria who underwent transplantation from June 2019 to October 2020 with grafts provided by Banc de Sang i Teixits' (BST) Cell Therapy Service were retrospectively included in the study and stratified in two groups regarding the use of cryopreserved grafts during the COVID-19 pandemic or fresh grafts in the previous time. Recipients were longitudinally followed until death or by day +100 after HSCT, whatever occurred first.

All patients were tested for SARS-CoV-2 by PCR before starting the conditioning procedure regardless of whether or not they presented with any symptoms. Donors were tested for SARS-CoV-2 prior to collection. Regarding UD HSC from countries where COVID-19 screening was not mandatory, a cryoquarantine of 14 days was required for these products by the Spanish Health Authority [12].

\section{Cryopreservation procedure}

For cryopreservation, leukapheresis material was concentrated prior to cryoprotection at $<300 \times 10^{6} \mathrm{TNC} / \mathrm{mL}$. The cryopreservation solution consisted of $10 \%$ dimethyl sulfoxide (DMSO), 4\% human serum albumin, and 5\% citrate dextrose solution A in Plasma-Lyte. A controlledrate/programmable freezer CM2010 (Carburos Medica, Barcelona, Spain) was used at $<-5{ }^{\circ} \mathrm{C} / \mathrm{min}$, and storage was performed at $<-150^{\circ} \mathrm{C}$ in vapor phase of liquid nitrogen. Three $0.5 \mathrm{~mL}$ aliquots of the cell preparation were frozen together with the bag and were used to perform the appropriate quality controls: blood cell count; flow cytometry assessment of CD45, CD34, and CD3 expression according to the ISHAGE protocol single-platform multigating strategy adjusting for forward side scatter, straight side scatter, CD45 and CD34 [13], and of viability using 7aminoactinomycin D (7-AAD); colony-forming unit (CFU) assay using Methocult 4434 from Stem Cell Technologies (Vancouver, Canada) supplemented with Iscove's Modified Dulbecco's Medium from BIOWEST (Nuaillé, France).

\section{Definitions}

Neutrophil recovery was considered to occur on the first of 3 consecutive days with an absolute neutrophil count $>500 /$ $\mu \mathrm{L}$ after the post-transplantation nadir. Platelet recovery date was the first of 3 consecutive days with a platelet count $>20,000 / \mu \mathrm{L}$, in the absence of platelet transfusion for 7 consecutive days. Full donor chimerism date was defined as the first day with complete donor chimerism (100\% donor) in total cells and/or peripheral cell subpopulations (CD15 and CD3), and was analyzed with a panel of 12 polymorphic short tandem repeat markers using the Mentype ${ }^{\mathrm{TM}}$ 
kit from Biotype (Dresden, Germany). Graft failure (GF) was defined as having never achieved absolute neutrophil count $>500 / \mu \mathrm{L}$, autologous recovery, or loss of donor engraftment. Acute GVHD was defined and graded according to standard criteria $[14,15]$. Preemptive therapy on CMV disease was considered when initiation of antiviral therapy for early asymptomatic CMV viremia detected by surveillance testing was started. PFS was defined as survival without progression or relapse of primary disease.

Available information about the reasons why some cryopreserved grafts were not finally used during the COVID-19 pandemic and until February 1, 2021 was registered, and serious adverse reactions (SAR) or events occurring in patients or in the procurement, respectively, were also recorded [16]. Regarding logistical issues any incident that happened throughout the process (cryopreservation, packaging, and transportation) was documented.

\section{Study endpoints}

The primary study endpoint was to compare hematological recovery (neutrophil and platelet engraftment) and full donor chimerism between patients who underwent UD HSCT with cryopreserved, non-manipulated PBSC during COVID-19 pandemic in Spain (March 13 to November 1, 2020) and those who had undergone the procedure with fresh non-manipulated PBSC in the immediately previous period (June 1, 2019 to March 12, 2020). Secondary endpoints included comparisons of acute GVHD, preemptive therapy on CMV disease, PFS, and OS between both cohorts. Finally, frozen grafts not transfused and logistical and clinical issues arising with cryopreserved UD HSC in the context of the pandemic were also assessed.

\section{Statistical analysis}

Two cohorts of UD HSCT were analyzed according to the use of fresh or cryopreserved products. Patient-, disease-, transplantation, and graft-related factors were compared between both groups using a comparison test (Mann-Whitney $U$ test for quantitative variables and $\chi^{2}$ or Fisher test for the categorical ones). Median and interquartile range (IQR) or frequency and percentage were used for quantitative and qualitative variables, respectively. Time to primary outcomes (neutrophil/platelet engraftment and $100 \%$ donor chimerism) and secondary outcomes (acute GVHD, preemptive therapy on CMV infection, PFS, and OS) were calculated using survival analyses. Kaplan-Meier curves and log-rank tests were used to compare both groups. A cause-specific analysis was performed for each individual outcome. Deaths without the event were treated as not-recoverable events. A type-I error of $5 \%$ was considered.

\section{Results}

\section{Baseline characteristics of patients and grafts}

A total of 47 UD HSC were cryopreserved during the COVID-19 pandemic. Forty-one patients underwent UD HSCT. Five and one patients were transplanted with grafts subjected to major $\left(\mathrm{CD} 34^{+}\right.$selection) and minor (washing or red cell depletion) manipulation, respectively, and bone marrow was used in three patients. All these nine patients were excluded from the clinical outcomes analysis. Therefore, 32 patients underwent UD HSCT with cryopreserved nonmanipulated PBSC during COVID-19 pandemic and were compared with 32 patients who had undergone UD HSCT with fresh non-manipulated PBSC in the immediately previous period. Results are summarized in Table 1 . There were no statistically significant differences between cohorts in terms of age, gender, hematologic malignancy, donor/recipient ABO, sex, HLA, or CMV serostatus compatibility. Conditioning, GVHD prophylaxis and prognosis index were comparable between two cohorts. Regarding graft characteristics (Table 1), no differences were observed between both groups except in the median number of $\mathrm{CD} 34^{+}$cells $/ \mathrm{kg}$ $\left(\times 10^{6}\right)$ prior to freezing or transfused in fresh, which was higher in those patients transplanted with cryopreserved grafts (6.0 versus $5.2 ; P=0.016$ ). The quality control in the postthawing reference samples of cryopreserved products is shown in Table 2. After cryopreservation, the median loss of viable $\mathrm{CD} 34^{+}$cells was $24.2 \%$. The median (IQR) of $\mathrm{CD} 45^{+} / 7$-AAD negative cells and $\mathrm{CD}^{+} / 7$-AAD negative cells were $66.8 \%(52.8-74.6)$ and $48.4 \%$ (33.7-64.8), respectively.

\section{Clinical outcomes}

Table 3 shows the univariate analysis of neutrophil/platelet engraftment, full donor chimerism, acute GVHD, preemptive therapy on CMV disease, PFS, and OS according to UD HSC. No statistical differences were found in the median days, the rate of recovery at day +21 and the cumulative incidence of neutrophil engraftment (Fig. 1A) between both groups. All patients transplanted with fresh grafts achieved neutrophil recovery within a median of 17.0 days versus 17.5 days in those transplanted with cryopreserved PBSC. In the group of patients transplanted with cryopreserved grafts, one of them reached neutrophil engraftment by day +33 and a herpes simplex virus 6 infection was detected. Another patient had GF in spite of an optimal cell dose, viability, and CFU growth. The median time to platelet recovery was 19.0 days with fresh grafts and 25.5 days with cryopreserved grafts $(P=0.192)$. No statistical differences were observed in the rate of platelet recovery at days +30 and +100 (Table 2), and cumulative incidence of platelet engraftment (Fig. 1B). All 
Table 1 Baseline characteristics of patients according to UD HSC.

\begin{tabular}{|c|c|c|c|}
\hline \multirow[t]{2}{*}{ Characteristic } & \multicolumn{2}{|l|}{ Cells } & \multirow[t]{2}{*}{$P$ value } \\
\hline & Fresh $(n=32)$ & $\begin{array}{l}\text { Cryopreserved } \\
(n=32)\end{array}$ & \\
\hline $\begin{array}{l}\text { Age at transplantation (years), median } \\
(I Q R)\end{array}$ & $50.5(32.2-61.2)$ & $52(38-66)$ & 0.333 \\
\hline Sex (female), $n(\%)$ & $11(34.4)$ & $(28.1)$ & 0.787 \\
\hline Disease, $n(\%)$ & & & 0.921 \\
\hline Acute leukemia ${ }^{a}$ & $17(53.1)$ & $16(50)$ & \\
\hline MDS/MPN & $6(18.8)$ & $7(21.9)$ & \\
\hline Lymphoma & $5(15.6)$ & $6(18.8)$ & \\
\hline Myeloma multiple & $4(12.5)$ & $2(6.2)$ & \\
\hline Chronic leukemia & $0(0)$ & $1(3.1)$ & \\
\hline$A B O D / R$ incompatibility, $n(\%)$ & $12(37.5)$ & $13(37.5)$ & 1.000 \\
\hline Sex D/R incompatibility, $n(\%)$ & $12(37.5)$ & $12(37.5)$ & 1.000 \\
\hline HLA compatibility, $n(\%)$ & & & 0.197 \\
\hline $9 / 10$ & $15(46.9)$ & $9(28.1)$ & \\
\hline $10 / 10$ & $17(53.1)$ & $23(71.9)$ & \\
\hline CMV D/R status, $n(\%)$ & & & 0.067 \\
\hline Pos/Pos & $20(62.5)$ & $10(31.2)$ & \\
\hline $\mathrm{Neg} / \mathrm{Pos}$ & $8(25)$ & $12(37.5)$ & \\
\hline $\mathrm{Neg} / \mathrm{Neg}$ & $2(6.2)$ & 7 21.9) & \\
\hline Pos/Neg & $2(6.2)$ & $3(9.4)$ & \\
\hline Myeloablative conditioning, $n(\%)$ & $14(43.8)$ & $14(43.8)$ & 1.000 \\
\hline GVHD prophylaxis, $n(\%)$ & & & 0.577 \\
\hline PTCy & $13(40.6)$ & $17(53.1)$ & \\
\hline Cyclosporine & $13(40.6)$ & $11(34.4)$ & \\
\hline Tacrolimus & $6(18.8)$ & $4(12.5)$ & \\
\hline$<90 \%$ Karnofsky Index, $n(\%)$ & $6(18.8)$ & $4(12.5)$ & 0.731 \\
\hline Disease Risk Index, $n(\%)$ & & & 0.308 \\
\hline Low & $4(12.5)$ & $3(9.4)$ & \\
\hline Intermediate & $15(46.9)$ & $21(65.6)$ & \\
\hline High & $8(25)$ & $7(21.9)$ & \\
\hline Very high & $5(15.6)$ & $1(3.1)$ & \\
\hline$H C T-C I, n(\%)$ & & & 0.125 \\
\hline 0 & $8(25)$ & $10(31.2)$ & \\
\hline $1-2$ & $11(34.4)$ & $15(46.9)$ & \\
\hline$>2$ & $13(40.6)$ & $7(21.8)$ & \\
\hline EBMT score, $n(\%)$ & & & 0.605 \\
\hline $0-2$ & $6(18.8)$ & $5(15.6)$ & \\
\hline $3-5$ & $22(68.8)$ & $20(62.5)$ & \\
\hline$>5$ & $4(12.5)$ & $7(21.9)$ & \\
\hline \multicolumn{4}{|l|}{ Graft characteristics } \\
\hline Number of days of collections, $n(\%)$ & & & 1 \\
\hline One & $29(90.6)$ & $30(93.8)$ & \\
\hline More than one & $3(9.4)$ & $2(6.2)$ & \\
\hline Hours from collection, median $(\mathrm{IQR})^{\mathrm{b}}$ & $36.2(29.4-48)$ & $41.9(29.7-59.4)$ & 0.327 \\
\hline \multicolumn{4}{|l|}{ Cellularity, median (IQR) ${ }^{\mathrm{c}}$} \\
\hline $\mathrm{TNC} \times 10^{8} / \mathrm{kg}$ & $6.4(5.1-8.7)$ & $6.3(5.1-7.5)$ & 0.682 \\
\hline Neutrophil $\times 10^{8} / \mathrm{kg}$ & $1.1(0.6-2.4)$ & $1.9(1.4-2.5)$ & 0.878 \\
\hline $\mathrm{CD} 34^{+} \times 10^{6} / \mathrm{kg}$ & $5.1(5-6)$ & $6(5.6-6)$ & 0.016 \\
\hline $\mathrm{CD}^{+} \times 10^{8} / \mathrm{kg}$ & $1.8(1.4-2.9)$ & $1.9(1.4-2.5)$ & 0.878 \\
\hline $\mathrm{TNC} / \mathrm{mL} \times 10^{6}$, median $(\mathrm{IQR})$ & $199.5(173.6-253.6)$ & $210.9(184.1-273.1)$ & 0.626 \\
\hline$\%$ mononuclear cells, median (IQR) & $80.6(73.9-85.3)$ & $83.7(78.4-90.3)$ & 0.155 \\
\hline \multicolumn{4}{|l|}{ Viability, median (IQR) } \\
\hline $\mathrm{CD} 45^{+} / 7-\mathrm{AAD}$ negative $(\%)$ & $98.7(97.8-99.1)$ & $99(98.2-99.2)$ & 0.205 \\
\hline CD34 ${ }^{+} / 7-\mathrm{AAD}$ negative $(\%)$ & $99.2(99-99.5)$ & $99.3(99-99.7)$ & 0.538 \\
\hline $\mathrm{CD}^{+} / 7$-AAD negative $(\%)$ & $98.6(96.9-99.2)$ & 99 (97.7-99) & 0.876 \\
\hline
\end{tabular}

UD HSC unrelated donor hematopoietic stem cell, IQR interquartile range, $M D S / M P N$ myelodisplastic syndrome/myeloproliferative neoplasm, $D / R$ donor/recipient, $H L A$ human leukocyte antigen, GVHD graft-versus-host disease, $P T C y$ post-transplantation cyclophosphamide, $H C T$-CI hematopoietic cell transplantation-specific comorbidity index, EBMT European Bone Marrow Transplantation, TNC total nucleated cell, 7-AAD 7-aminoactinomicine D.

${ }^{a}$ Four and eight patients with acute lymphoblastic leukemia in fresh group and cryopreserved group, respectively. Thirteen and eight patients with acute myeloblastic leukemia in fresh group and cryopreserved group, respectively.

${ }^{\mathrm{b}}$ To transfusion (fresh cells) or prior cryopreservation (cryopreserved cells).

cIn cells to be transfused to fresh group patients ("Fresh" column), or, prior to cryopreservation, in cells to be transfused to cryopreserved group patients ("Cryopreserved" column).

survivors achieved platelet engraftment by 100 days after the procedure. Regarding full donor chimerism, no statistical differences were found in the median days, the rate of achievement at day +30 and +100 , and the cumulative incidence between both cohorts (Table 2 and Fig. 1C). One and two patients in each group who received fresh and cryopreserved grafts, respectively, achieved mixed chimerism by 100 days after transplantation. All three patients had received RIC conditioning.

The cumulative incidence of acute GVHD (Table 2 and Fig. 2A) observed at day +100 in patients transplanted with fresh cells was $31 \%$ versus $41 \%$ in those transplanted with cryopreserved cells $(P=0.380)$. No statistical differences were observed in incidence of preemptive therapy on CMV between both groups (Table 2 and Fig. 2B).

The rates of PFS at day +100 were $81 \%$ (95\% confidence interval [CI] [69-96]) and 88\% (95\% CI [77-100]) in fresh and cryopreserved grafts, respectively $(P=0.500)$ (Table 2 and Fig. 2C). The corresponding rates of OS at day +100 were $81 \%$ (95\% CI [69-96]) and 90\% (95\% CI [90-100]) $(P=0.300)$ (Table 2 and Fig. 2D).

\section{Clinical and logistical issues in cryopreserved grafts}

Six of $47(13 \%)$ donated and cryopreserved UD HSC had not yet been transplanted at February 1, 2021. The main cause was relapse/progression of underlying disease (three cases). The others cases corresponded to a patient's HLAtyping error that was detected after collection, a patient who presented with DSA refractory to desensitization treatment and finally, and one patient who refused HSCT days after graft cryopreservation. The median (IQR) number of days that elapsed from collection to transplantation was 21 (21-49). In one case, cryopreserved cells were transfused 175 days after collection.

Only one SAR was documented at transfusion of HPC. That patient had tachycardia and generalized cutaneous rash, because of the incompatibility between the external cryopreserved donor bag and the transfusion set, which forced healthcare staff to transfuse urgently the cells manually, which precluded an appropriate transfusion speed control. An allergic reaction to DMSO was suspected, which was completely resolved after antihistamine treatment and decreasing transfusion rate. 
Neither patients nor donors had a SARS-CoV-2 positive test.

The median hours from collection to transfusion or cryopreservation was 36.2 with fresh PBSC and 41.9 with frozen PBSC, respectively $(P=0.327$ ) (Table 1$)$. Such delay was due to the policy of TC to perform in-house cryopreservation. Nevertheless, seven UD HSC were cryopreserved at collection centers because of the unavailability of in-fresh transportation. In one case, this onsite cryopreservation was unintended and led to a deviation of procedures that complicated and delayed quality release of the product for transplantation albeit with no impact on clinical outcomes. In another case, rapid instructions to package the cryopreserved product had to be issued to an external facility and, in a third case, a problem caused by the incompatibility size between BST's cell processing laboratory cassettes and the rack provided by the sender within the dry shipper had to be overcome.

Table 2 Quality parameters of cryopreserved cells.

\begin{tabular}{lc}
\hline Quality test & Median (IQR) \\
\hline $\mathrm{CD} 45^{+} / 7-\mathrm{AAD}(\%)$ & $66.8(52.8-74.6)$ \\
$\mathrm{CD} 34^{+} / 7-\mathrm{AAD}(\%)$ & $81.8(66.0-94.35)$ \\
$\mathrm{CD} 3^{+} / 7-\mathrm{AAD}(\%)$ & $48.4(33.7-64.8)$ \\
$\mathrm{CFU}-\mathrm{GM} / \mathrm{kg} \times 10^{5}$ & $9.0(5.8-13.8)$ \\
Total CD34 ${ }^{+}$recovery $(\%)$ & $96.2(80.8-100)$ \\
Viable CD34 $^{+}$recovery $(\%)$ & $75.8(60.5-88.4)$ \\
TNC recovery $(\%)$ & $100(99.2-100)$ \\
\hline
\end{tabular}

7-AAD 7-aminoactinomicine $\mathrm{D}, C F U$-GM granulocyte/macrophage colony-forming units, $T N C$, total nucleated cell.

\section{Discussion}

In spite of the fact that reliable randomized, controlled studies regarding graft cryopreservation impact on clinical outcomes of patients undergoing UD HSCT were not available when the COVID-19 pandemic broke out, cryopreservation was recommended as a general rule. The aim of our study was to assess the impact of this recommendation comparing engraftment and clinical outcomes of patients who underwent UD HSCT with cryopreserved nonmanipulated PBSC during COVID-19 pandemic with those who had undergone this procedure with fresh nonmanipulated PBSC in the previous period. Also, we reviewed the unused frozen grafts, and clinical and logistical issues regarding cryopreservation. Our results suggest that cryopreservation during the COVID-19 pandemic did not negatively affect neutrophil/platelet engraftment and full donor chimerism. In order to prevent engraftment delays or failures, a negative impact of insufficient $\mathrm{CD} 34^{+}$cell dose on survival outcomes, and cell losses or low viability due to cryopreservation, the amount of frozen $\mathrm{CD} 34^{+}$cells was intentionally higher than that transfused in fresh grafts $[17,18]$. Regarding quality control of cryopreserved grafts, all the UD HSC were fully compliant according to cell counts, viability and CFUs. CD45 ${ }^{+} / 7-\mathrm{AAD}$ and $\mathrm{CD} 3^{+} / 7-$ AAD negative cells were lower than $70 \%$ due to the impact of cryopreservation, but this did not influence potency assays. One GF occurred in the cohort transplanted with cryopreserved PBSC. However, the quality control showed that cell dose, purity, and potency were optimal. Time from collection to cryopreservation was $4 \mathrm{~h}$ and the patient
Table 3 Univariate analysis of neutrophil/platelet engraftment, full donor chimerism, acute GVHD, preemptive therapy on cytomegalovirus disease, PFS, and OS according to UD HSC.

\begin{tabular}{|c|c|c|c|}
\hline \multirow[t]{2}{*}{ Variable } & \multicolumn{2}{|l|}{ Cells } & \multirow[t]{2}{*}{$P$ value } \\
\hline & Fresh $(N=32)$ & Cryopreserved $(N=32)$ & \\
\hline \multicolumn{4}{|l|}{ Neutrophils } \\
\hline Days to recovery, median $(95 \% \mathrm{CI})$ & $17(14-24)$ & $17.5(16-21)$ & 0.767 \\
\hline Rate of recovery at 21 days $(95 \% \mathrm{CI})$ & $72(51-84)$ & $75(54-86)$ & 0.850 \\
\hline \multicolumn{4}{|l|}{ Platelets } \\
\hline Days to recovery, median (95\% CI) & $19(17-35.5)$ & $25.5(19-31)$ & 0.192 \\
\hline Rate of recovery at 30 days $(95 \% \mathrm{CI})$ & $69(48-81)$ & $69(47-81)$ & 0.620 \\
\hline Rate of recovery at 100 days $(95 \% \mathrm{CI})$ & $94(76-98)$ & $97(76-96)$ & 0.620 \\
\hline \multicolumn{4}{|l|}{ Full donor chimerism } \\
\hline Days to achievement, median (95\% CI) & $31.5(27-56.5)$ & $35(26-48.2)$ & 0.872 \\
\hline Rate of achievement at 30 days $(95 \% \mathrm{CI})$ & $44(24-59)$ & $25(8-39)$ & 0.990 \\
\hline Rate of achievement at 100 days $(95 \% \mathrm{CI})$ & $75(54-86)$ & $84(65-93)$ & 0.990 \\
\hline Rate of acute GVHD at 100 days $(95 \%$ CI) & $31(13-46)$ & $41(21-55)$ & 0.380 \\
\hline Rate of preemptive CMV at 100 days $(95 \%$ CI) & $38(18-52)$ & $38(18-52)$ & 1.000 \\
\hline Rate of PFS at 100 days $(95 \%$ CI) & $81(69-96)$ & $88(77-100)$ & 0.500 \\
\hline OS at 100 days $(95 \%$ CI) & $81(69-96)$ & $90(85-100)$ & 0.300 \\
\hline
\end{tabular}

$C I$ confidence interval, $G V H D$ graft-versus-host disease, $C M V$ cytomegalovirus, $P F S$ progression-relapsefree survival, $O S$ overall survival. 

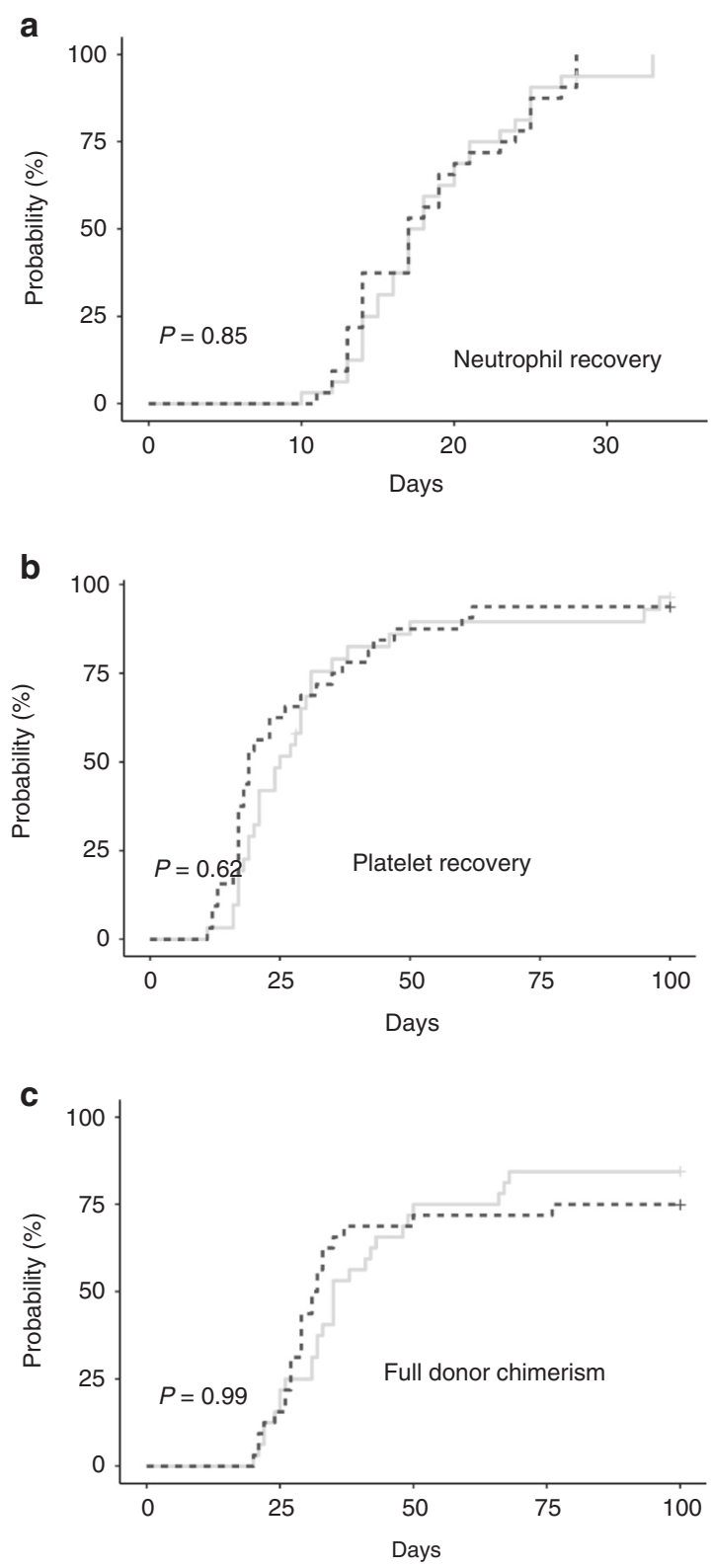

Fig. 1 Cumulative incidence of hematological recovery and full donor chimerism according to UD HSC (cryopreserved versus fresh). The cumulative incidences of A neutrophil recovery, B platelet recovery, and $\mathbf{C}$ full donor chimerism are shown for the groups of patients who underwent UD HSCT with either cryopreserved (solid line) or fresh PBSC (dashed line).

received RIC conditioning. Risk factors for GF other than cryopreservation condition have been described [19]. Both cohorts were comparable regarding acute GVHD, preemptive therapy on CMV disease, PFS, and OS at 100 days.

The impact on clinical outcomes of using cryopreserved grafts for HSCT procedures is controversial. Our findings contrast with some studies using frozen PBSC, which concluded that cryopreservation and subsequent transportation of PBSC may impair their engraftment in allogeneic HSCT [10].
However, other studies are in line with ours $[5,9,11]$. Interestingly, one study showed that the outcomes of patients who underwent HSCT with cryopreserved or fresh PBSC were similar in terms of neutrophil and platelet recovery, recurrence, PFS, and OS, although the study focused on MRD HSCT [9]. Our results are also in agreement with those of a recent report stating that cryopreservation of either MRD or UD allografts does not delay hematopoietic recovery, increase the risk of GVHD and PFS, or decrease OS after HSCT using GVHD prophylaxis with PTCy [5]. Furthermore, our finding that cryopreservation was associated with no impact on survival had already been reported in patients who underwent HSCT with UD HSC [11].

Although cryopreservation has undoubted advantages such as securing graft integrity before the start of conditioning, our study has also elicited some warnings. Six cryopreserved grafts were donated, transported, and cryopreserved, but not finally used, which means that $13 \%$ of donations remained stored by the end of the study. Interestingly, a study recently carried out by DKMS stem cell donor registry concluded that $5-10 \%$ of cryopreserved products will not be eventually transfused [20]. The requirement of quarantine for the donations received from countries where SARS-CoV-2 tests were not mandatory could has increased the odds of relapse or progress among recipients, which in fact happened in three cases, who thus lost their status as HSCT candidates [12]. A corrective policy could be scheduling transplantation and collection very closely in order to proceed with patient conditioning within a few days of UD HSC cryopreservation, time enough for performing mandatory quality controls. Furthermore, one product had to be discarded due to an HLA-typing error in the first HLA test. The HLA confirmatory result was obtained before the patient started conditioning, but, unfortunately, after donor collection. This kind of inconvenience may appear when work is performed under unbearable pressure, in conditions such as those created by COVID-19. This argument prompted us to adopt changes to ensure rigorous quality assurance with risk assessment when contingency plans are activated and cryopreservation is recommended for all products.

Finally, unintended transportation issues occasionally led to cryopreservation of grafts at origin, which resulted in lastminute decisions. One undesired consequence was that one external cryopreserved bag caused an allergic reaction by DMSO due to manual transfusion at an inappropriate rate. There were also packaging issues that might have compromised the safety of HSC. To prevent these problems, the coordination among collection centers, cell processing centers, and TCs must improve, and logistical issues concerning shipment and delivery must be anticipated. Moreover, we consider that the development of international guidelines by reference professional societies addressing the logistics of cryopreservation and transportation is urgently needed for adaption and harmonization with external centers and beyond. 

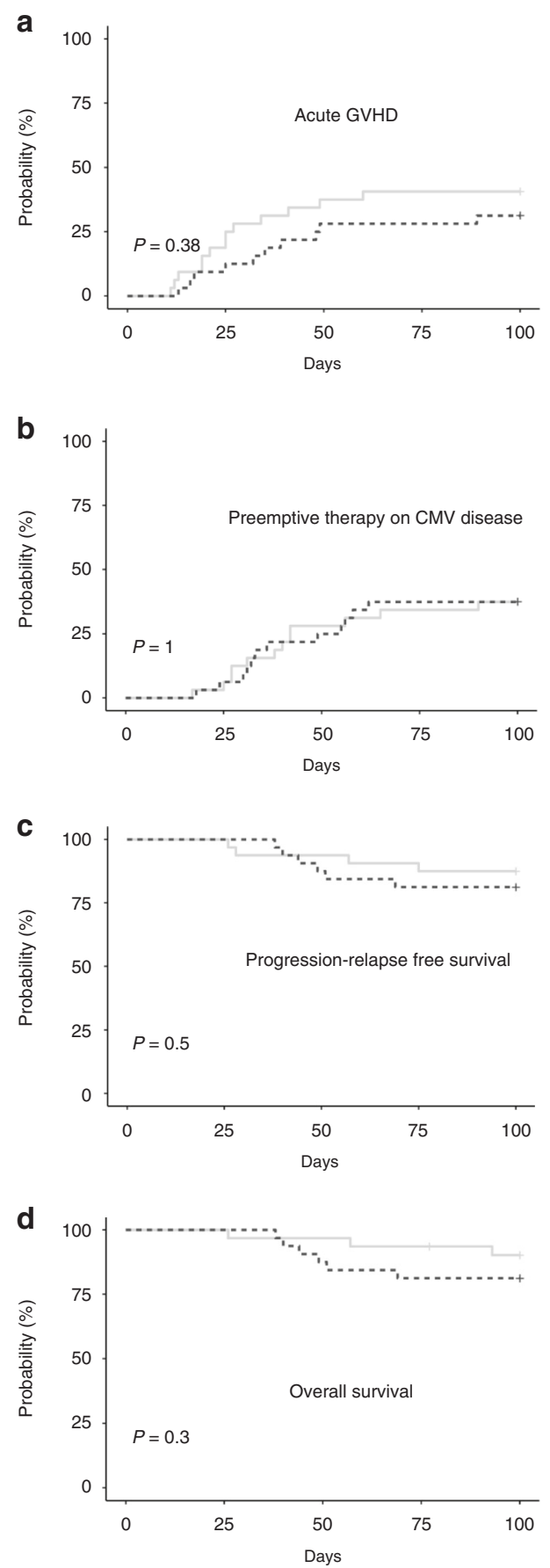

Fig. 2 Cumulative incidence of acute GVHD and preemptive CMV treatment disease, PFS, and OS according to UD HSC (cryopreserved versus fresh). The cumulative incidences of $\mathbf{A}$ acute graftversus-host disease (GVHD), B preemptive therapy on CMV disease, $\mathbf{C}$ progression-relapse-free survival, and $\mathbf{D}$ overall survival are shown for the groups of patients who underwent UD HSCT with either cryopreserved (solid line) or fresh grafts (dashed line).

Our study has limitations: sample size of the cohorts was limited by the short duration of the recruitment period and the follow-up period was also necessarily short. Grafts subjected to major and minor manipulation, as well as BM grafts, were excluded from the clinical outcomes analyses. Therefore, long-term effects of cryopreservation as well as the influence of manipulation or using BM as graft source must be addressed in future studies.

In summary, to the best of our knowledge, this is the first study to assess the impact of cryopreservation on UD transplantation outcomes during the COVID-19 pandemic. Since no statistical differences were found regarding engraftment, GVHD, preemptive CMV therapy, PFS, and OS, we consider that cryopreservation was a safe decision to maintain the UD HSCT program in TCs. However, further studies are needed. Importantly, wasting donations raises ethical issues, and logistic incidents that could affect product quality or timely delivery must be avoided. For this purpose, improvements in disaster plans led by international guidelines and harmonization in cryopreservation requirements are urgently needed in these complex scenarios. More efforts are required to ensure that all cryopreserved UD HSC are eventually transfused.

Acknowledgements We want to thank Jesus Maroto, Silvia Tuset, and Mar Sanchez for their administrative support, and Ramón Montes for his writing support. We also want to acknowledge BST Cell Therapy Service technical team (Sonia Maroto, Maria Ruz, Yolanda Chacon, Sergio Arteaga, Marina Hortola, and Camila Cardona) and cellular laboratory (Javier Algar, Begoña Amill, Margarita Blanco, Ruth Forner, Daniel Navarro, Aroa Perez, and Isabel Tarrago) for their effort, and external cell processing laboratories (Adoración Hernandez from Blood and Tissue Bank of Granada, Raquel de Paz from Hospital Universitario La Paz, Luis Larrea from Blood and Tissue Bank of Valencia, and Olga Lopez from Hospital Universitario de Salamanca) and external TC (Jaime Sanz from Hospital Universitari La Fe de Valencia). We also thank donors, patients, couriers, Spanish and other registries, and everyone who contributed to make UD HSCT possible in these difficult times. Statistical analysis has been carried out in the Statistics and Bioinformatics unit (UEB) Vall d'hebron Hospital Researh Institue (VHIR).

\section{Compliance with ethical standards}

Conflict of interest The authors declare no competing interests.

Publisher's note Springer Nature remains neutral with regard to jurisdictional claims in published maps and institutional affiliations.

\section{References}

1. Frey NV, Lazarus HM, Goldstein SC. Has allogeneic stem cell cryopreservation been given the "cold shoulder"? An analysis of the pros and cons of using frozen versus fresh stem cell products in allogeneic stem cell transplantation. Bone Marrow Transpl. 2006;38:399-405.

2. Szer J, Weisdorf D, Querol S, Foeken L, Madrigal A. The impact of COVID-19 on the provision of donor hematopoietic stem cell products worldwide: collateral damage. Bone Marrow Transpl. 2020;23:1-2.

3. Recommendations of the European Society for Blood and Marrow Transplantation (EBMT) regarding the situation generated by the COVID-19 pandemic. 2020. https://www.ebmt.org/sites/default/files/ 
2020-03/EBMT\%20COVID-19\%20guidelines\%20v.4.3\%20\% 282020-03-23\%29.pdf.

4. Recommendations of the World Marrow Donor Association (WMDA) regarding donor medical suitability in the situation generated by the COVID-19 pandemic 2021. https://share.wmda.info/pa ges/viewpage.action?pageId $=344866320$.

5. Hamadani M, Zhang MJ, Tang XY, Fei M, Brunstein C, Chhabra $\mathrm{S}$, et al. Graft cryopreservation does not impact overall survival after allogeneic hematopoietic cell transplantation using posttransplantation cyclophosphamide for graft-versus-host disease prophylaxis. Biol Blood Marrow Transpl. 2020;26:1312-7.

6. Eckardt JR, Roodman GD, Boldt DH, Clark GM, Alvarez R, Page $\mathrm{C}$, et al. Comparison of engraftment and acute GVHD in patients undergoing cryopreserved or fresh allogeneic BMT. Bone Marrow Transpl. 1993;11:125-31.

7. Stockschläder M, Hassan HT, Krog C, Krüger W, Löliger C, Horstman $\mathrm{M}$, et al. Long-term follow-up of leukaemia patients after related cryopreserved allogeneic bone marrow transplantation. Br J Haematol. 1997;96:382-6.

8. Shinkoda Y, Ijichi O, Tanabe T, Ishikawa S, Kamitamari A, Nishikawa $\mathrm{T}$, et al. Identical reconstitution after bone marrow transplantation in twins who received fresh and cryopreserved grafts harvested at the same time from their older brother. Clin Transpl. 2004;18:743-7.

9. Kim DH, Jamal N, Saragosa R, Loach D, Wright J, Gupta V, et al. Similar outcomes of cryopreserved allogeneic peripheral stem cell transplants (PBSCT) compared to fresh allografts. Biol Blood Marrow Transpl. 2007;13:1233-43.

10. Lioznov M, Dellbrugger C, Sputtek A, Fehse B, Kroger N, Zander AR. Transportation and cryopreservation may impair haematopoietic stem cell function and engraftment of allogeneic PBSCs, but not BM. Bone Marrow Transpl. 2008;42:121-8.

11. Medd P, Nagra S, Hollyman D, Craddock C, Malladi R. Cryopreservation of allogeneic PBSC from related and unrelated donors is associated with delayed platelet engraftment but has no impact on survival. Bone Marrow Transpl. 2013;48:243-8.

12. Update of October 5, 2020, of the guidelines of the Spanish National Transplant Organization (Spain Ministry of Health,
Consumer Affairs and Social Welfare) about the impact of SARSCoV-2 and COVID-19 on donation and transplantation, reference BV-ES-20200122-11. http://www.ont.es/infesp/Paginas/COVID19.aspx.

13. Lanza F, Saccardi R, Seghatchian J. New horizons on stem cell cryopreservation through the artificial eyes of CD34 using modern flow cytometry tools. Transfus Apher Sci. 2020;59: 102785.

14. Przepiorka D, Weisdorf D, Martin P, Klingemann HG, Beatty P, Hows J, et al. 1994 consensus conference on acute GVHD grading. Bone Marrow Transpl. 1995;15:825-8.

15. Jagasia MH, Greinix HT, Arora M, Williams KM, Wolff D, Cowen EW, et al. National Institutes of Health consensus development project on criteria for clinical trials in chronic graft-versus-host disease: I. The 2014 Diagnosis and Staging Working Group report. Biol Blood Marrow Transpl. 2015;21:389-401. e1.

16. Shaw BE, Chapman J, Fechter M, Foeken L, Greinix H, Hwang $\mathrm{W}$, et al. Towards a global system of vigilance and surveillance in unrelated donors of haematopoietic progenitor cells for transplantation. Bone Marrow Transpl. 2013;48:1506-9.

17. Mattsson J, Ringdén O, Storb R. Graft failure after allogeneic hematopoietic cell transplantation. Biol Blood Marrow Transpl. 2008; $14: 165-70$.

18. Torlen J, Ringden O, Le Rademacher J, Batiwalla M, Chen J, Erkers $\mathrm{T}$, et al. Low CD34 dose is associated with poor survival after reduced-intensity conditioning allogeneic transplantation for acute myeloid leukemia and myelodysplastic syndrome. Biol Blood Marrow Transpl. 2014;20:1418-25.

19. Olsson RF, Logan BR, Chaudhury S, Zhu X, Akpek G, Bolwell $\mathrm{BJ}$, et al. Primary graft failure after myeloablative allogeneic hematopoietic cell transplantation for hematologic malignancies. Leukemia. 2015;29:1754-62.

20. Schmidt AH, Buk D, Platz A, van den Brink MRM. Cryopreservation for all is no option in unrelated stem cell transplantation. Comment on Dholaria B, et al. securing the graft during pandemic: are we ready for cryopreservation for all? Biol Blood Marrow Transpl. 2020;26:e145-6. 